\title{
Pathophysiology of human lipoprotein receptors: clinical consequences of a cellular defect
}

\author{
JAMES SHEPHERD, CHRISTOPHER J PACKARD \\ From the University Department of Biochemistry, Royal Infirmary, Glasgow G4 OSF
}

SUMMARY The function of the low density lipoprotein receptor has been reviewed at the cell level and in man. Its key role in cholesterol metabolism is unquestioned and it is central to the actions of a number of important hypocholesterolaemic agents. Clearly it must be involved in atherogenesis since its dysfunction leads to premature and severe atherosclerosis in both animals and man. The tools are now available to address this question and answers should be forthcoming in the near future.

All normal cells share a fundamental ability to gauge their requirement of specific nutrients, to select the appropriate substances from the thousands of potential candidates in their environment, and to assimilate them in a regulated fashion. These processes depend critically upon the operation of membrane receptors which allow the cell to interact with its environment to its own benefit and (in the case of multicellular creatures) to that of the organism as a whole. Receptors come in many varieties. Some like the well studied acetylcholine receptor, participate in short range intercellular communication while others are capable of detecting humoral signals from distant sites and respond to them by modulating cellular metabolism. Typical examples of the latter are the receptors for insulin, prolactin and epidermal growth factor. Besides participating as signal transducers, many receptors have a primary role in facilitating the uptake of specific nutrients from the environment. Often, their dysfunction is lethal in utero or leads soon after birth to the kinds of serious clinical complications seen in victims of the Fanconi syndrome and cystinuria.

Recently, the nature of a more subtle and insidious receptor defect has been uncovered. ${ }^{1}$ In this condition, known as familial hypercholesterolaemia, a specific aberration in the gene regulating the production of membrane receptors responsible for cellular cholesterol assimilation leads first to hypercholesterolaemia and subsequently to severe and premature atherosclerosis. The unravelling of the properties of this low density lipoprotein receptor pathway has provided valuable insight into the important role played by receptors in the cellular

Accepted for publication 5 October 1983 economy. In the following discussion we will focus attention on the low density lipoprotein receptor pathway and emphasise in particular the metabolic and clinical consequences which result from its dysfunction.

\section{Receptor mediated endocytosis}

Vesicular transport occurs in all eukaryotic cells. It is responsible for cellular import (endocytosis) and export (exocytosis) of nutrients and other substances and also shuttles cell constituents between organelles. ${ }^{2}$ The cytoplasmic vacuoles generated in the process, though adopting a wide variety of shapes and sizes, share the property of being encapsulated in a limiting membrane which comes from the organelle of origin by a budding process. Upon arrival at their destination, they discharge their contents by fusing with the membrane of the recipient compartment. This system transports a prodigious quantity of material and so there is a heavy demand for membrane components. For example, every hour the plasma membrane of the macrophage internalises the equivalent of twice its entire surface area as endocytic vesicles. ${ }^{3}$ Kinetic studies have shown that synthesis alone cannot match this flux rate and it is believed that recycling of the various membranes must occur, so that the vesicle envelope is returned to its site of origin. ${ }^{1}$ Processes, at present poorly understood, prevent randomisation of the membrane components ${ }^{4}$ and maintain the unique structure and function of each organelle.

Endocytosis is the generic term which describes the import of low molecular weight solutes and macromolecules by the cell. ${ }^{5}$ It embraces a variety of activities which include (a) ingestion of particulate material, with the formation of a phagosome and (b) 
the continuous imbibing (pinocytosis) of pericellular fluid. In the latter, substances are assimilated at their environmental concentration, which for many nutrients is low. On this basis the process is inefficient, non-selective, and unregulated. However, there are specialised areas of the plasma membrane where controlled and specific uptake does occur. They are characterised by being coated on their cytoplasmic aspect with regularly spaced "bristles" of clathrin. ${ }^{467}$ At these sites, certain plasma membrane proteins are clustered while others are excluded. ${ }^{4}$ Many of the former are receptors synthesised by the cell for the purpose of appropriating specific ligands from the environment and concentrating them at this particular area of the cell membrane. ${ }^{8}$ It then undergoes rapid endocytosis generating a highly structured vesicle coated on its exterior with clathrin and on its luminal surface with the ligand bound to the receptor protein embedded in the membrane. This mechanism displays two important features which derive from the participation of specific membrane proteins. First, assimilation is highly selective since receptor-ligand interaction is analogous to the lock-and-key arrangement which exists between an enzyme and its substrate. And secondly, since the availability of the receptor protein can be controlled, the whole process is amenable to regulation. Both of these properties characterise the endocytosis of cholesterol which occurs via the agency of the low density lipoprotein receptor pathway. ${ }^{9}$

The low density lipoprotein receptor pathway in cultured cells

The basic features of the low density lipoprotein receptor pathway in cultured cells (Fig. 1) are now established, largely as a result of the pioneering and sustained investigations of Brown, Goldstein and their collaborators in Dallas, Texas. ${ }^{89}$

The receptor, which has now been isolated, is an acid glycoprotein ${ }^{10}$ with a molecular weight in the range 100-120 kilodaltons. ${ }^{11}$ After synthesis in the rough endoplasmic reticulum it becomes glycosylated in the Golgi apparatus and is thereafter inserted randomly into the cytoplasmic membrane where it moves spontaneously to bristle-coated regions. ${ }^{13}$ This migration, at least in the case of low density lipoprotein, occurs even before the receptor binds the ligand so that at any one time up to $70 \%$ of the low density lipoprotein receptor protein on the membrane is localised in these specialised areas which occupy no more than $2 \%$ of the cell membrane surface. ${ }^{68}$ Electron microscopic studies suggest that these regions continuously become indented and pinch off to form coated vesicles surrounded by a bristly network of clathrin. ${ }^{6}$ The frequency of this event is such that the average low density lipoprotein receptor remains on the cell membrane for only a few minutes before being internalised as a component of the coated vesicle. Notwithstanding, most of them in this short time specifically acquire low density lipoprotein by an electrostatic process critically dependent on $\mathrm{Ca}^{++}$ ions ${ }^{9}$ and involving the interaction between arginine and lysine residues on the lipoprotein and negative domains on the receptor itself. ${ }^{13}$ Following endocytosis, the coated vesicle with its low density lipoprotein charge bound on the luminal surface to receptor proteins rapidly sheds its clathrin coat and migrates towards lysosomes, fusing with other uncoated endocytic vesicles on the way. During migration, the intravesicular $\mathrm{pH}$ decreases and this may be

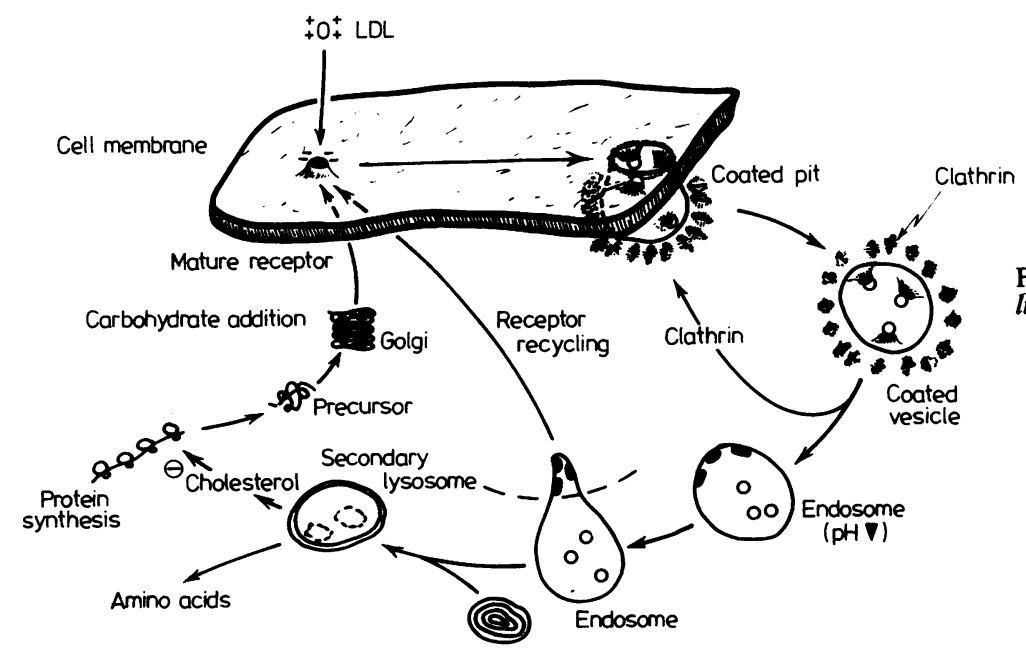

Fig. 1 The autoregulated low density lipoprotein receptor pathway. 
Mutant alleles at the low density lipoprotein receptor locus. In this schema the various forms of the low density lipoprotein receptor protein are referenced according to their apparent molecular weights on sodium dodecyl sulphate gels. The wild type precursor receptor protein migrates with an apparent molecular weight of 120 kilodaltons while the mature product has a migration rate equivalent to that of a protein of 160 kilodaltons.

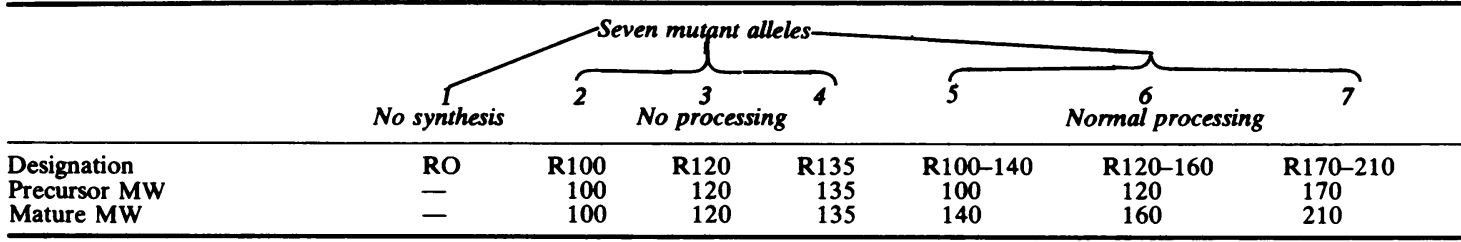

$\mathrm{n}=77 \mathrm{FH}$ homozygotes

From Tolleshaug $\mathrm{H}$, et al. ${ }^{17}$ The LDL receptor locus in familial hypercholesterolaemia: multiple mutations disrupt transport and processing of a membrane receptor. Cell 1983;32:941-51.

responsible for promoting the dissociation of low density lipoprotein from its receptor. ${ }^{12}$ The latter returns to the plasma membrane in a recycling process which has obvious economic benefits in that the average low density lipoprotein receptor is capable of being used more than 150 times in its 30-hour lifespan. ${ }^{12}$ The receptor-depleted vesicle transports its low density lipoprotein centrally towards the lysosomes by a mechanism which is facilitated by microtubular elements of the cell cytoskeleton. ${ }^{14}$ Upon arrival, both organelles fuse, thereby delivering the lipoprotein to the digestive actions of lysosomal hydrolases. Cholesterol esters, the major lipid components of the particles, are cleaved, releasing the free sterol into the cell. It triggers three key regulatory responses. ${ }^{9}$ First, synthesis of 3-hydroxy-3-methylglutaryl coenzyme A reductase (HMG CoA reductase), the rate limiting microsomal enzyme responsible for cholesterol production is reduced, diminishing cholesterologenesis within the cell. Secondly, another microsomal enzyme, acyl coenzyme A: cholesterol acyltransferase (ACAT), is activated causing excess sterol to be reesterified and stored as cholesteryl ester (oleate) droplets. And, thirdly, the sterol released by lysosomal hydrolysis suppresses continued synthesis of the low density lipoprotein receptor protein, limiting further endocytic uptake of low density lipoprotein. So, all three responses act in concert to protect the cell from the toxic effects of overaccumulation of the sterol. They also serve to balance the availability of environmental cholesterol with the cell's requirement for this lipid, maintaining a constant level of the sterol in the face of fluctuations in the external supply. ${ }^{15}$ When none is available, the cells continue to grow by synthesising it for themselves. Conversely, if low density lipoprotein is present in the pericellular fluids, the cells suppress endogenous sterol sythesis and use their receptors preferentially to assimilate it from their environment.
Molecular anatomy of the receptor protein

Early measurements of low density lipoprotein receptor activity on fibroblasts from patients with the clinical and biochemical stigmata of homozygous familial hypercholesterolaemia indicated that the condition was heterogeneous. Three functional classes could be identified on the basis of the cell culture studies." Some individuals were "receptor negative", their fibroblasts containing less than $2 \%$ of the normal number of active receptors. In others, receptor activity was at a level of $2-30 \%$ of normal. These were classified as being "receptor defective." And a few individuals turned out to have an "internalisation defect". ${ }^{16}$ Their fibroblasts, surprisingly, bound a normal complement of low density lipoprotein to their surfaces but thereafter failed to translocate the lipoprotein inside the cell for lysosomal digestion. This functional classification has now been complemented by a structural classification system. The key to this was the purification by the Dallas group of low density lipoprotein receptor protein from bovine adrenal cortex. ${ }^{10}$ This was used to prepare an antireceptor antibody which cross reacted with the normal human fibroblast receptor. Armed with this probe, Tolleshaug et $a l^{17}$ then proceeded to make a detailed immunological examination of their collection of fibroblasts grown from 77 familial hypercholesterolaemic homozygotes from around the world. First, they screened the receptor negative cells (which failed to bind low density lipoprotein) for the presence of membrane receptor antigen and found that $40 \%$ were positive. Obviously these cultures were still able to manufacture the protein, but it was ineffective. Their attention then turned to probing the details of the mechanism responsible for the synthesis and membrane insertion of the receptor. Preliminary studies with normal fibroblasts showed that the active receptor is synthesised in a two stage process. Following a pulse with $\left[{ }^{35} \mathrm{~S}\right]$ methionine, radioactivity was rapidly incorporated into an immunologically detectable cytoplasmic 
receptor protein with a nominal molecular weight of 120 kilodaltons. Within $30-45 \mathrm{~min}$ of synthesis, this protein became glycosylated and for the first time appeared on the cell surface membrane. Thus, its synthesis occurs by a two stage process and the receptor appears on the cytoplasmic membrane only in its mature glycosylated form. When kinetic studies of this kind were performed on the 77 available homozygous familial hypercholesterolaemic cultures a pattern of results emerged which is outlined in the Table. At least seven different forms of the low density lipoprotein receptor seem to exist and family studies support the view that these derive from seven mutations. In the first (group 1, Table), precursor synthesis failed. These cells bound neither low density lipoprotein nor the antireceptor antibody. Cells in groups 2,3 and 4 did synthesise a cytoplasmic precursor protein but it was defective in that its molecular weight varied and it failed to mature and be inserted into the cytoplasmic membrane. So, again their membranes failed to bind low density lipoprotein and did not interact with the antireceptor antibody. The last three groups of cell cultures $(5,6$ and 7 , Table) were capable of producing a receptor with the capacity to mature in the cytoplasm and be inserted into the cell membrane, as measured by their ability to interact with the anti receptor antibody. However, they failed to bind and internalise low density lipoprotein competently.

All of the structural phenotypes depicited in the Table seem to derive from mutations at the genetic locus for the low density lipoprotein receptor. The evidence in favour of this conclusion comes from examination of fibroblasts from family members (particularly parents) of the propositus. Most familial hypercholesterolaemic "homozygotes" happen, in fact, from cell culture studies of their parents fibroblasts, to be double heterozygotes, acquiring one mutant allele from one parent and a different one from another. In all of the studies described, no case arose in which a mutant allele from one parent could compensate in the propositus for a different mutation from the second, as might have been expected had, for example, the defect been in a gene coding for a processing or membrane insertion protein. On this basis, the available evidence supports the contention that there are at least seven mutant alleles at the low density lipoprotein receptor locus. Further studies are required to establish the molecular basis of each of these.

The low density lipoprotein receptor pathway in man As far as the cell is concerned, the low density lipoprotein receptor pathway is simply an autoregulated transport mechanism for cholesterol. In the context of the intact animal, the pathway operates not only to regulate intracellular sterol levels but also plays a critical role in maintaining corporeal cholesterol homeostasis. ${ }^{18}$ In this situation, its malfunction can have serious consequences for those cells susceptible to damage by high concentrations of the sterol. Such a phenomenon is considered by many investigators to be an important contributor to atherosclerosis.

The clinical significance of the low density lipoprotein receptor pathway first became apparent during investigations of subjects suffering from the condition known as familial hypercholesterolaemia. This inherited disease, transmitted in autosomal dominant fashion, is characterised by severe hypercholesterolaemia, extravascular tendinous and cutaneous sterol deposits particularly in areas subject to trauma, and accelerated and premature vascular disease. ${ }^{19}$ Patients heterozygous for the condition commonly have plasma cholesterol concentrations which are 2-3 times normal and often develop symptoms of coronary artery insufficiency by the fifth decade. The course of events in "homozygotes" (vide supra) is even more rapid, and quite distinct. Their hypercholesterolaemia is marked (plasma cholesterol concentrations reach values 6-7 times normal) and evident at birth. Unique subcutaneous xanthomatous cholesterol deposits may also be present in the newborn and certainly develop within the first five years of life; and very early evidence of generalised atherosclerosis rapidly appears, usually leading to death before the age of 30 . Measurement of low density lipoprotein receptor activity in fibroblasts cultured from the skin of these individuals gave the first clue to the aetiology of their problem, and suggested that the defect lay in expression of the receptor for this lipoprotein. In the heterozygous fibroblasts, only half of the normal receptor activity was expressed, while in "homozygous" cells, receptor activity was absent or barely detectable.

Goldstein and Brown were the first to appreciate from the above evidence that the receptor plays a pivotal role in balancing intra- and extracellular cholesterol metabolism. They hypothesised that where the receptor pathway was inefficient, the burden of cholesterol clearance would fall on less well controlled, potentially damaging alternative catabolic mechanisms. ${ }^{20}$ The postulation of two distinct metabolic pathways for low density lipoprotein catabolism led to a search for ways of quantifying their contributions to the process. The procedure which evolved ${ }^{21}$ was based on the observation that the electrostatic interaction between low density lipoprotein and its receptor could be abolished by chemical modification of the arginine or lysine residues on the lipoprotein apoprotein. ${ }^{13}$ Treatment with 1,2 cyclohexanedione which blocks the arginine residues, abolishes the in vitro interaction of low 
density lipoprotein with the receptors on cultured fibroblasts. When this modified tracer was injected into the bloodstream, its plasma clearance was delayed. The magnitude of the delay provided an index of the contribution made by the receptor pathway to low density lipoprotein catabolism as a whole. ${ }^{22}$ Other alternative procedures which modify the lysine residues on low density lipoprotein can be used in a similar way. ${ }^{2324}$ In young healthy volunteers we found that the receptor pathway was responsible for about half of low density lipoprotein degradation..$^{22} 25$ So, the alternative catabolic mechanism(s) were significant in vivo. Their functions and location is not known, although several possibilities have been considered. For example, all cells in the body participate in fluid pinocytosis which clears a constant proportion of the extracellular fluid each day. Its contribution will not exceed the catabolic rate of albumin and therefore must be less than about $5 \%$ of the plasma pool per day. So, even in receptor deficient familial hypercholesterolaemic "homozygotes" (whose total low density lipoprotein clearance is about $12-15 \%$ of the plasma pooi per day) ${ }^{26}$ pinocytosisis not the major clearance mechanism. One must therefore consider alternative possibilities such as the involvement of the reticuloendothelial system. Macrophages found in the tissues of familial hypercholesterolaemic "homozygotes" have certainly been shown to accumulate low density lipoprotein cholesterol,' acquiring in the process a typical foamy appearance. Moreover, suppression of reticuloendothelial activity in vivo retards receptor-independent low density lipoprotein catabolism and raises the circulating level of the lipoprotein. ${ }^{27}$ But as yet the extent of its involvement and the way in which it operates is unknown.

When native and arginine modified tracers of low density lipoprotein were injected into subjects suffering from familial hypercholesterolaemia it was obvious that the balance between receptormediated and receptor-independent low density lipoprotein catabolism had shifted. Heterozygotes cleared only half the normal amount of low density lipoprotein by the receptor route ${ }^{21}$ consistent with the finding that their cells express a reduced receptor complement in culture. In the "homozygotes" who were studied, the native and modified tracers were catabolised at identical rates. ${ }^{28} 29$ So, in these individuals, receptor activity was not detectable and the burden of low density lipoprotein clearance was shifted entirely into alternative receptorindependent routes. The deficiency of receptor activity detectable in their fibroblasts in vitro is associated with reduced or absent receptor mediated clearance of low density lipoprotein in vivo and results in accumulation of the lipoprotein in the plasma of these individuals. Moreover, their susceptibility to atherosclerosis tends to implicate the receptor-independent low density lipoprotein clearance mechanisms in the pathogenesis of the disease. Which particular mechanism or mechanisms are responsible for the pathological cholesteryl ester accumulation in the arterial wall is unknown. Histological studies have shown that two distinct cell types, smooth muscle cells and macrophages, predominate in the lesions. The question at the forefront of many current studies is how these cells accumulate low density lipoprotein in an uncontrolled fashion. In atherosclerosis induced in rabbits by dietary cholesterol supplementation we have obtained evidence ${ }^{30}$ to suggest that in the lesion receptors escape their normal control and continue to take up low density lipoprotein when they should have been suppressed. Other workers have invoked alternative possibilities, ${ }^{31}$ including modification to the structure of low density lipoprotein, to explain the avidity with which cells in the lesion assimilate the lipoprotein.

The double isotope procedure for measuring low density lipoprotein receptor activity in vivo has stimulated one other important development. For the first time, it has become possible to examine the flexibility of the pathway in the intact organism and this has led to studies designed to assess the receptor response to pharmacological and surgical manoeuvres aimed at lowering plasma cholesterol concentrations. These revealed the important role which the liver plays in modulating plasma low density lipoprotein. This was surprising in light of the prevailing opinion that the organ had little to do with low density lipoprotein catabolism. However, with hindsight its involvement is almost obligatory since only the liver has the capacity to excrete the sterol or its derivatives the bile acids in appreciable amounts; and in fact the organ occupies a central place in two related but distinct sterol transporting pathways $^{32}$ - the enterohepatic circulation on the one hand and systemic lipoprotein metabolism on the other (Fig. 2). These systems are linked by a regulatory hepatocyte cholesterol pool which, according to the principles described earlier for the cultured fibroblast model, modulates the activity of low density lipoprotein receptors in the liver.

At present, the drugs of choice for the treatment of hypercholesterolaemia are the bile acid sequestrant resins (cholestyramine and colestipol). They are neither digested in nor absorbed from the gut but act there to bind bile acids and interrupt their return to the liver via the enterohepatic circulation..$^{32}$ In consequence, the hepatic conversion of cholesterol to bile acids rises, draining the hepato- 


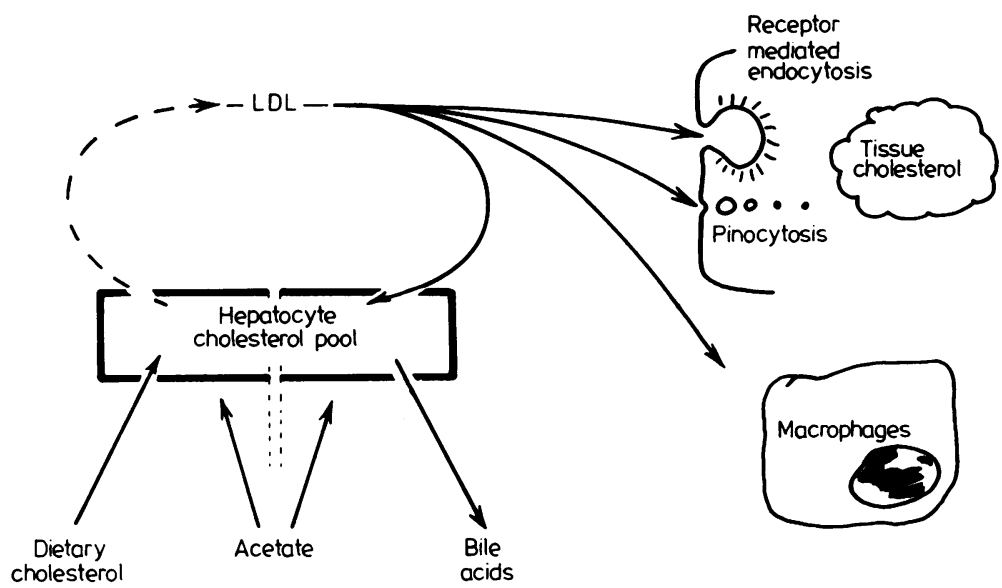

Fig. 2 Low density lipoprotein metabolism and cholesterol transport in vivo. Low density lipoprotein is the major cholesterol transporting vehicle in human plasma. It is derived from larger triglyceride-rich lipoproteins synthesised in the liver and is catabolised throughout the body by a number of mechanisms, one of which depends on a high affinity cell membrane receptor. The regulatory hepatocyte cholesterol pool is fed by dietary sterol and de novo synthesis. Its depletion occurs during bile acid production. Kinetic evidence suggests that the pool is compartmented.

cyte sterol pool. Simultaneously, there is a fall in the level of plasma low density lipoprotein, which results from an increase in its rate of catabolism. ${ }^{29} \mathrm{In}$ particular, the receptor pathway is activated, ${ }^{29}$ and animal studies have demonstrated that receptors in the liver are responsible for this effect. ${ }^{33} 34$ That is, the organ responds to an increased demand for the sterol by turning on the receptors and drawing upon the plasma low density lipoprotein pool. So, it is possible to activate the pathway even in heterozygous familial hypercholesterolaemic subjects whose problem derives from a partial defect of receptor expression. Ileal bypass surgery provides an alternative and effective means of interrupting the enterohepatic circulation. Here, a short circuit is constructed round the terminal ileum which is the major site of bile acid reabsorption. The effect on circulating low density lipoprotein concentrations is even more striking than that achieved by sequestrant resins and the mechanism behind it seems to be the same. ${ }^{35}$ But, as might be predicted, "homozygotes" are refractory both to resin therapy and ileal bypass unless they belong to that group of "receptor defective" individuals who retain a residuum of receptor function. ${ }^{32}$ "Receptor negative" homozygotes have lost the vital link between the systemic and enterohepatic circulations and in response to sequestrant therapy will convert grams of hepatogenous cholesterol to bile acids without altering plasma low density lipoprotein levels.

So far, attention has been restricted to procedures designed to increase hepatic cholesterol utilisation. An equally viable alternative would be to interrupt its synthesis, leading to a depletion of the regulatory sterol pool, activation of the low density lipoprotein receptor mechanism and, presumably, a fall in circulating low density lipoprotein cholesterol. Recently, Endo et $a^{36}$ isolated a potent competitive inhibitor of HMG CoA reductase from the culture broth of the mould Penicillium citrinum. This drug, Compactin, and its close relative, Mevinolin, ${ }^{37}$ obtained from Aspergillus terreus are potent competitive inhibitors of sterol synthesis. Both effectively lower low density lipoprotein cholesterol levels in control and heterozygous familial hypercholesterolaemic subjects ${ }^{38}$ by increasing, as predicted, the receptor mediated clearance of the lipoprotein from the plasma; ${ }^{39}$ and when a bile acid sequestrant resin is added to the regimen a much greater decrease in low density lipoprotein levels is obtained than can be achieved by either agent alone. ${ }^{39}$ Since one blocks hepatic cholesterol synthesis and the other potentiates its utilisation it is predictable that their synergistic actions are directed in concert towards the activation of hepatic low density lipoprotein receptors, a prediction borne out by animal studies. ${ }^{34}$ So, we are now able by these manoeuvres to redirect low density lipoprotein catabolism to the liver and reduce the amount of cholesterol cleared by receptor-independent catabolic routes. The above therapeutic regimens therefore redress the imbalance between receptor-dependent and 
receptor-independent low density lipoprotein catabolism.

One intriguing and as yet unexplained feature of the response to the above pharmacological and surgical interventions is that only catabolism was affected. Synthesis of the lipoprotein remained constant ${ }^{29} 39$ even though it is produced in the liver by a process which requires cholesterol. Therefore, the hepatocyte does not appear to economise at the expense of low density lipoprotein secretion at times when there is a heavy demand on the organ's sterol pool. One potential explanation ${ }^{32}$ for this is that intracellular hepatic sterol pools are compartmentalised (Fig. 2). It is quite possible that the cholesterol destined for lipoproteins is partitioned from that which is converted to bile acids.

Fig. 2 depicts a third mechanism whereby the hepatic cholesterol pool can be perturbed. Dietary cholesterol in the form of chylomicron remnants transfer the sterol rapidly from gut to liver, leading to an expansion of the hepatic sterol pool ${ }^{40}$ According to the receptor hypothesis, this should suppress hepatic low density lipoprotein receptor activity, a phenomenon which has recently been noted in chylomicron infused dogs. ${ }^{41}$ We have examined the situation in man using cholesterol supplemented diets. ${ }^{25}$ The results were more complex than predicted. Both synthesis and catabolism changed, but the former had the greater impact, and was primarily responsible for the $40 \%$ increase in plasma low density lipoprotein levels. The fall in catabolism was attributable to the receptor pathway but was less than the receptor hypothesis led us to expect. Two factors probably contribute to this. First, dietary sterol was being shunted into the systemic circulation; and secondly, it may be that the adult human liver normally expresses few low density lipoprotein receptors. ${ }^{42}$ Under these circumstances, their suppression would therefore have little effect on total plasma low density lipoprotein clearance. When the results of this study are considered in light of the proven relationship between dietary cholesterol intake and atherosclerosis it is noteworthy that the diet-induced rise in plasma low density lipoprotein resulted in more sterol being channelled into receptor-independent catabolic pathways. There seems no doubt that the latter merit further study as potential contributors to the process of atherogenesis.

The authors are indebted to Annette Fox for her secretarial expertise. This work was completed during the tenure of grants from the Scottish Home and Health Department (K/MRS/50/C429), the Medical Research Council (G8111558SA) and the Cancer Research Campaign (SP 1671).

\section{References}

${ }^{1}$ Goldstein JL, Brown MS. Familial hypercholesterolemia: pathogenesis of a receptor disease. Johns Hopkins Med J 1978;143:8-16.

${ }^{2}$ Palade GE. Problems in intracellular membrane traffic. In: Evered D, Collins GM, eds. Membrane recycling. Ciba Foundation Symposium 92. London: Pitman Books Ltd, 1982:1-14.

${ }^{3}$ Steinman RM, Brodie SE, Cohn ZA. Membrane flow during pinocytosis. A stereologic analysis. J Cell Biol 1976;68:65587.

4 Bretscher MS, Thomson JN, Pearse BMF. Coated pits as molecular filters. Proc Natl Acad Sci USA 1980;77:4156-9.

s Silverstein SC, Steinman RM, Cohn ZA. Endocytosis. Ann Rev Biochem 1977;46:669-722.

- Anderson RGW, Brown MS, Goldstein JL. Role of the coated endocytic vesicle in the uptake of receptor bound low density lipoprotein in human fibroblasts. Cell 1977;10:351-64.

' Pearse BMF. On the structural and functional components of coated vesicles. J Mol Biol 1978;126:803-12.

- Goldstein JL, Anderson RGW, Brown MS. Coated pits, coated vesicles and receptor-mediated endocytosis. Nature 1979;279:679-85.

9 Goldstein JL, Brown MS. The low density lipoprotein pathway and its relation to atherosclerosis. Ann Rev Biochem 1977;46:897-930.

${ }^{10}$ Schneider WJ, Beisiegel U, Goldstein JL, Brown MS. Purification of the low density lipoprotein receptor, an acidic glycoprotein of 164000 molecular weight. J Biol Chem 1982;257:2664-73.

"Tolleshaug H, Goldstein JL, Schneider WJ, Brown MS. Posttranslational processing of the LDL receptor and its genetic dysruption in familial hypercholesterolemia. Cell 1982;30:715-24.

${ }^{12}$ Brown MS, Anderson RGW, Goldstein JL. Recycling receptors: the round trip itinerary of migrant membrane proteins. Cell 1983;32:663-7.

${ }^{13}$ Mahley RW, Innerarity TL, Weisgraber KH. Alterations in metabolic activity of plasma lipoproteins following selective chemical modification of the apoproteins. Ann $N$ Y Acad Sci 1980;348:265-80.

${ }^{14}$ Ostlund RE, Pfleger B, Schonfeld G. Role of microtubules in low density lipoprotein processing by cultured cells. J Clin Invest 1979;63:75-84.

is Brown MS, Goldstein JL. General scheme for regulation of cholesterol metabolism in mammalian cells. In: Dietschy JM, Gotto AM, Outko JA, eds. Disturbances in lipid and lipoprotein metabolism. Bethesda: American Physiological Society, 1978:183-90.

${ }^{16}$ Goldstein JL, Brown MS. The LDL receptor locus and the genetics of familial hypercholesterolemia. Ann Rev Genetics 1979;13:259-89.

17 Tolleshaug H, Hobgood KK, Brown MS, Goldstein JL. The LDL receptor locus in familial hypercholesterolemia: multiple mutations disrupt transport and processing of a membrane receptor. Cell 1983;32:941-51.

${ }^{18}$ Packard CJ, Shepherd J. Low-density lipoprotein receptor pathway in man: Its role in regulating plasma low-density lipoprotein levels. Atheroscler Rev 1983;11:29-63.

19 Goldstein JL, Brown MS. Familial hypercholesterolemia. In: Stanbury JB, Wyngaarden JB, Fredrickson DS, Goldstein JL, Brown MS, eds. The metabolic basis of inherited disease 5th ed. New York: McGraw-Hill, 1982;672-712.

${ }^{20}$ Goldstein JL, Brown MS. Atherosclerosis: The low density lipoprotein receptor hypothesis. Metabolism 1977;26:125675.

${ }^{21}$ Shepherd J, Bicker S, Lorimer AR, Packard CJ. Receptor mediated low density lipoprotein catabolism in man. $J$ Lipid Res 1979;20:999-1006.

${ }^{22}$ Slater HR, Packard CJ, Shepherd J. Measurement of receptor 
independent lipoprotein catabolism using 1,2 cyclohexanedione modified low density lipoprotein. $J$ Lipid Res 1982;23:92-6.

${ }^{23}$ Kesaniemi YA, Witztum JL, Steinbrecher UP. Receptor mediated catabolism of low density lipoprotein in man. Quantitation using glucosylated low density lipoprotein.J Clin Invest 1983;71:950-9.

${ }^{24}$ Slater HR, McKinney L, Shepherd J, Packard CJ. Receptor independent low density lipoprotein catabolism: Evaluation of 2-hydroxyacetaldehyde treated lipoprotein as a probe for its measurement. Biochim Biophys Acta 1984; (in press).

${ }^{25}$ Packard CJ, McKinney L, Carr K, Shepherd J. Cholesterol feeding increases low density lipoprotein synthesis. J Clin Invest 1983;72:45-51.

${ }^{26}$ Bilheimer DW, Stone NJ, Grundy SM. Metabolic studies in familial hypercholesterolemia. Evidence for a gene dosage effect in vivo. J Clin Invest 1979;64:524-33.

${ }^{27}$ Slater HR, Packard CJ, Shepherd J. Receptor independent catabolism of low density lipoprotein: Involvement of the reticuloendothelial system. J Biol Chem 1982;257:307-10.

${ }^{28}$ Thomson GR, Soutar AK, Spengel FA, Jadhav A, Gavigan SJJP, Myant NB. Defects of receptor mediated low density lipoprotein catabolism in homozygous familial hypercholesterolemia and hypothyroidism in vivo. Proc Natl Acad Sci USA 1981;78:2591-5.

${ }^{29}$ Shepherd J, Packard CJ, Bicker S, Lawrie TDV, Morgan HG. Cholestyramine promotes receptor mediated low density lipoprotein catabolism. $N$ Engl J Med 1980;302:1219-22.

${ }^{30}$ Slater HR, Shepherd J, Packard CJ. Receptor mediated catabolism and tissue uptake of human low density lipoprotein in the cholesterol fed atherosclerotic rabbit. Biochim Biophys Acta 1982;713:435-45.

${ }^{31}$ Henricksen T, Mahoney EM, Steinberg D. Enhanced macrophage degradation of low density lipoprotein previously incubated with cultured endothelial cells: Recognition by receptors for acetylated low density lipoproteins. Proc Natl Acad Sci USA 1981;78:6499-503.

${ }^{32}$ Packard CJ, Shepherd J. The hepatobiliary axis and lipoprotein metabolism. J Lipid Res 1982;23:1081-98.

${ }^{33}$ Slater HR, Packard CJ, Bicker S, Shepherd J. Effects of cholestyramine on receptor mediated plasma clearance and tissue uptake of human low density lipoprotein in the rabbit. $J$ Bio Chem 1980;255:10210-3.

${ }^{34}$ Kovanen PT, Bilheimer DW, Goldstein JL, Jaramillo JJ, Brown MS. Regulatory role for hepatic low density lipoprotein receptors in vivo in the dog. Proc Natl Acad Sci USA 1981;78:1194-8.

3s Spengel FA, Jadhav A, Duffield RGM, Wood CB, Thomson GR Superiority of partial ileal bypass over cholestyramine in reducing cholesterol in familial hypercholesterolaemia. Lancet 1981 ;ii:768-70.

${ }^{36}$ Endo A, Kuroda M, Tsujita Y. ML-236A, ML-236B and ML236C, new inhibitors of cholesterologenesis produced by Penicillium citrinum. J Antibiot (Tokyo) 1976;29:1346-8.

${ }^{37}$ Alberts A, Chen W, Kuron J, et al. Mevinolin, a highly competitive inhibitor of hydroxymethylglutaryl Coenzyme A reductase and a cholesterol lowering agent. Proc Natl Acad Sci USA 1980;77:3957-61.

${ }^{38}$ Mabuchi H, Haba T, Tatami R, et al. Effects of an inhibitor of 3-hydroxy-3-methylglutaryl coenzyme A reductase on serum lipoproteins and Ubiquinone 10 levels in patients with familial hypercholesterolemia. $N$ Engl J Med 1981;305:478-82.

${ }^{39}$ Bilheimer DW, Grundy SM, Brown MS, Goldstein JL. Mevinolin and colestipol stimulate receptor mediated clearance of low density lipoprotein from plasma in familial hypercholesterolemia heterozygotes. Proc Natl Acad Sci USA 1983;80:4124-8.

${ }^{40}$ Dietschy JM, Wilson JD. Regulation of cholesterol metabolism. $N$ Engl J Med 1970;282:1128-38, 1179-83, 1241-9.

${ }^{41}$ Angelin B, Ravioli CA, Innerarity TL, Mahley RW. Regulation of hepatic lipoprotein receptors in the dog. Rapid regulation of apolipoprotein B, E receptors but not of apolipoprotein E receptors by intestinal lipoproteins and bile acids. J Clin Inves 1983;71:816-31.

${ }^{42}$ Mahley RW, Hui DY, Innerarity TL, Weisgraber KH. Two independent lipoprotein receptors on hepatic membranes of dogs swine and man. J Clin Invest 1981;68:1197-206.

Requests for reprints to: Dr J Shepherd, Department of Biochemistry, Royal Infirmary, Glasgow G4 OSF, Scotland. 